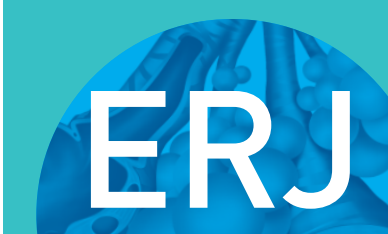

open research
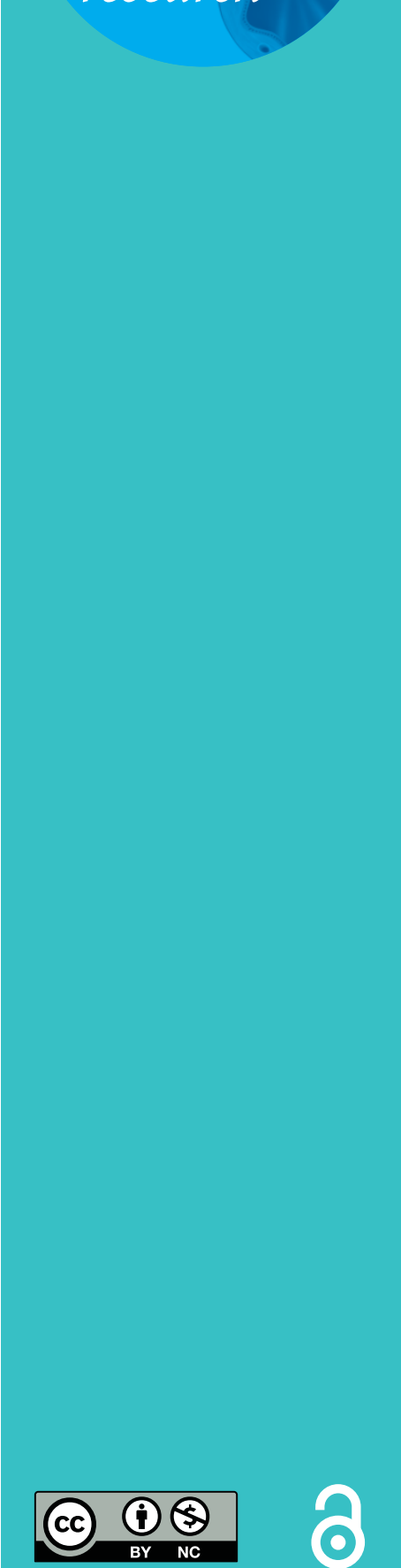

\section{Antibiotic preferences for childhood pneumonia vary by physician type and European region}

To the Editor:

Antibiotics are the most commonly prescribed medicines for acutely unwell children worldwide [1]. Many of these antibiotic prescriptions are issued in a primary care setting [2-4]. Despite this, a robust evidence base for agent selection in primary care is lacking for many childhood indications, including community-acquired pneumonia (CAP). In an era of increasing antibiotic resistance, with optimal antibiotic use being paramount to preserve this precious resource, trials involving ambulatory patients representative of those seen in primary care are needed to address this gap.

29 randomised controlled trials involving 14188 children with CAP were included in an updated Cochrane meta-analysis to identify effective antibiotics for this indication [5]. The authors note that 1) robust head-to-head comparisons of frequently used antibiotic options for otherwise healthy children are lacking and 2) meta-analysis findings are not likely to be generalisable to settings with low CAP mortality, such as high-income countries and primary care [5].

There are three key guidance documents that include recommendations about antibiotic therapy for ambulatory patients in high-income settings: the British Thoracic Society (BTS) and the Infectious Diseases Society of America (IDSA) guidelines, and the European Society for Paediatric Infectious Diseases (ESPID) discussion document on CAP. These all recommend amoxicillin, an extended-spectrum penicillin, as first-line oral treatment for this indication in mild-moderate disease (as often seen in primary care) regardless of age [6-8]. Both the BTS and IDSA guidelines specify that their recommendations apply only to otherwise healthy children $[6,7]$.

Macrolides, amoxicillin/clavulanate and cephalosporins are also noted as potential treatment options by all three guidelines, with macrolides commonly being indicated for older children with signs and symptoms compatible with atypical pathogens [6-8]. Amoxicillin/clavulanate and cephalosporins are specifically recommended for children with incomplete pneumococcal or Haemophilus influenzae type b vaccination status by ESPID and IDSA. These three antibiotics have a broader spectrum of coverage, and could have greater adverse effects on the human microbiome and more unwanted effects for treated patients, including the selection of resistant bacteria [9-11].

In the USA, high (around 20\%) but falling rates of cephalosporin use have been observed [2,3]. In the UK, penicillins (including amoxicillin/clavulanate) and macrolides are the most commonly prescribed antibiotics for the treatment of children in primary care [4]. Given the observed variation in ambulatory care antibiotic prescribing for childhood CAP, selecting the optimal agent remains a key unresolved question, which could be appropriately addressed in a pragmatic randomised trial. In line with recommendations on research into CAP management in low-mortality settings, such a trial would need to be designed as a noninferiority trial with a focus on patient-relevant outcomes [12]. However, such a strategic (and likely large) trial is only of value if agent selection is indeed an important dilemma for prescribers.

We aimed to determine and compare preferred first-line antibiotic options for treatment of childhood CAP amongst general practitioners (GPs) and primary care paediatricians (PPs), the main primary care providers for children in Europe, to assess which comparators may be relevant in a noninferiority trial of antibiotics for this indication.

A targeted, cross-sectional online survey was carried out amongst 1574 members of the European Academy of Paediatrics Research in Ambulatory Settings network (EAPRASnet) (www.eapaediatrics.eu) in July 2014. The survey comprised five focussed questions. It was also distributed during the winter season 2014-2015 to 200 GPs through the Platform for European Preparedness Against (Re-emerging) Epidemics (PREPARE) (www.prepare-europe.eu).

The survey asked respondents to report their first-line antibiotic choices for treatment of CAP in otherwise healthy children up to 5 years old (younger group) and those $>5$ years (older group) who can be managed at home after primary care assessment. Descriptive statistics were calculated using STATA 13.1 (StataCorp, 
College Station, TX, USA). Differences between GPs and PPs as well as regional differences (respondents grouped according to the United Nations geoscheme for the European region [13]) were evaluated using $\chi^{2}$ testing.

The EAPRASnet response rate was 46.4\% (n=730; 528 PPs, 54 GPs and 148 others) with 163 additional responses from the PREPARE network (150 GPs, six PPs and seven others), providing data from 738 primary care providers for analysis.

A preference for aminopenicillins or narrow-spectrum penicillins was expressed by 463 (63\%) out of 738 respondents for treatment of younger children and by 280 (38\%) out of 736 for older children. For older children, macrolides were frequently prescribed in the first line of treatment (271 (37\%) out of 736). The second most common antibiotic used in the first line for younger children was amoxicillin/clavulanate (208 (28\%) out of 738)

GPs had a somewhat stronger preference for first-line aminopenicillins than PPs (for younger children, $64 \%$ versus $55 \%(\mathrm{p}=0.02)$; for older children, $55 \%$ versus $25 \%(\mathrm{p}<0.001))$; PPs had a noticeably stronger preference for first-line macrolides than GPs when treating older children $(46 \%$ versus $11 \%(\mathrm{p}<0.001)$ ). Cephalosporins were reported as being used first-line in $2 \%$ of younger children and $1 \%$ of older children by GPs, and in $7 \%$ of children regardless of age by PPs.

Table 1 shows the responses according to region and practitioner type. A stronger aminopenicillin or penicillin preference was observed for GPs than for PPs in Eastern and Western Europe for both age groups, and for treatment of older children among GPs in Southern and Northern Europe. In addition, substantial regional variations were observed; for example, only $28 \%$ of PPs in Eastern Europe preferred aminopenicillin treatment for younger children compared with 78\% in Northern Europe.

Overall, aminopenicillin or penicillin treatment was the preferred option for the majority of children of all ages in Northern Europe (91\% for younger children and $84 \%$ for older children) and Western Europe (61\% and $45 \%$, respectively). In Eastern Europe, amoxicillin/clavulanate was most frequently indicated as being used first-line for younger (45\%) and older children (38\%). In Southern Europe, aminopenicillins or penicillins were preferred for younger children (67\%) and macrolides for older children (47\%). Macrolides were also the second most commonly reported first-line option for older children in Western Europe (33\%).

Our highly focused, rapid survey indicates the level of clinical equipoise amongst primary care physicians regarding different antibiotic treatment options in childhood CAP. While the survey was distributed to two research networks, and therefore did not reach the majority of nonresearcher primary care providers, the participants are practitioners likely to be involved in recruitment into a potential trial. Consequently, the acceptability of the trial comparators to these practitioners is paramount. Furthermore, it is unlikely that the children seen by practitioners within these networks differ from children seen by other primary care providers. Prescribing preferences for children with underlying disease cannot be determined from the

TABLE 1 Comparison of number of general practitioners (GPs) and primary care

paediatricians (PPs) expressing a preference for first-line prescribing of amoxicillin or

narrow-spectrum penicillins to children with community-acquired pneumonia by European

region

\begin{tabular}{lcccc} 
Practitioner type & \multicolumn{2}{c}{ Region } \\
& Northern Europe & Eastern Europe & Southern Europe & Western Europe \\
\hline $\begin{array}{l}\text { 5 } \text {-year-olds } \\
\text { PP }\end{array}$ & $9(77.8,48.0-97.2)$ & $86(27.9,18.8-38.7)$ & $323(66.9,61.4-72.0)$ & $116(50.0,40.6-59.4)$ \\
GP & $61(93.4,84.1-98.2)$ & $41(51.2,35.1-67.1)$ & $52(69.2,54.9-81.3)$ & $50(88.0,75.7-95.5)$ \\
P-value & 0.168 & 0.01 & 0.737 & $<0.001$ \\
$>5$-year-olds & & & & \\
PP & $9(55.6,21.2-86.3)$ & $86(15.1,8.3-24.5)$ & $323(30.0,25.1-35.4)$ & $116(28.4,20.5-37.6)$ \\
GP & $60(88.3,77.4-95.2)$ & $40(32.5,18.6-49.1)$ & $52(48.1,34.0-62.4)$ & $50(82.0,68.6-91.4)$ \\
P-value & 0.030 & 0.025 & 0.01 & $<0.001$
\end{tabular}

Data are presented as $\mathrm{n}(\%, 95 \%$ Cl) unless otherwise stated. Northern Europe: Denmark, Finland, Ireland, Lithuania, Norway, Sweden and the UK; Eastern Europe: Albania, the Czech Republic, Hungary, Romania and Slovakia; Southern Europe: Cyprus, Spain, Greece, Croatia, Israel, Italy, Montenegro, Malta, Portugal and Slovenia; Western Europe: Austria, Belgium, Switzerland, Germany, France, Luxembourg and the Netherlands. 
survey, which focused specifically on otherwise healthy children. Additional factors influencing antibiotic selection, such as immunisation status, were not elicited. The response rate for the EAPRASnet survey was just below 50\%. Given that the survey was open for only 3 weeks and no reminders were sent, participation was high. However, it is possible that nonrespondents would have indicated different prescribing preferences. Finally, we were unable to ascertain actual prescribing practices, which may differ from responses to our survey.

We were able to highlight the heterogeneity of selecting antibiotics for childhood CAP between different types of clinicians and across different regions of Europe. While both GPs and PPs express an aminopenicillin preference for CAP treatment of children of all ages, amoxicillin/clavulanate and macrolides are also frequently prescribed first-line. Potentially reflecting the greater role of atypical pathogens in older children $[7,8]$, macrolides were preferred in some settings for children $>5$ years of age. Such variation in current practice needs to be considered during the design of international trial protocols; for example, when considering comparators relevant to front-line clinical practice. Notably, the results demonstrate the likely inappropriateness of cephalosporins as a childhood CAP trial comparator choice in Europe, despite being listed as a secondary treatment option in available guidance.

Julia A. Bielicki ${ }^{1,2}$, Charlotte I.S. Barker ${ }^{1}$, Alike W. van der Velden ${ }^{3}$, Mike Sharland ${ }^{1}$, Diego van Esso ${ }^{4}$, Adamos Hadjipanayis ${ }^{5}$, Stefano del Torso ${ }^{6}$ and Zachi Grossman ${ }^{7}$

${ }^{1}$ Paediatric Infectious Diseases Research Group, Institute for Infection and Immunity, St George's, University of London, London, UK. ${ }^{2}$ Paediatric Pharmacology, University of Basel Children's Hospital, Basel, Switzerland. ${ }^{3}$ Julius Center for Health Sciences and Primary Care, University Medical Center Utrecht, Utrecht, The Netherlands. "Primary Care Center "Pare Claret", Catalan Institute of Health, Barcelona, Spain. ${ }^{5}$ Medical Faculty, European University of Cyprus, Nicosia, Cyprus. ${ }^{6}$ Studio Pediatrico Vecellio 33, ULSS 16, Padua, Italy. ${ }^{7}$ Maccabi Health Services, Tel Aviv, Israel.

Correspondence: Julia A. Bielicki, Paediatric Infectious Diseases Research Group, Institute for Infection and Immunity, St George's University of London, Cranmer Terrace, London, UK. E-mail: jbielick@sgul.ac.uk

Received: Jan 112016 | Accepted after revision: April 022016

Support statement: No financial or material support was received for this work. J.A. Bielicki is funded as a Clinical Research Fellow by the European Union Seventh Framework Programme project PREPARE (Grant Agreement number 602525). C.I.S. Barker is funded as a Clinical Research Fellow by the Global Research in Paediatrics Network of Excellence, part of the European Union's Seventh Framework Programme for research, technological development and demonstration (under Grant Agreement number 261060). Funding information for this article has been deposited with FundRef.

Conflict of interest: Disclosures can be found alongside this article at openres.ersjournals.com

Acknowledgements: We would like to thank all EAPRASnet and PREPARE participants who completed the survey. We are also grateful to Lucy Yardley (University of Southampton, Southhampton, UK) for providing critical input into the survey design.

@ERSpublications

Survey of EAPRASnet and @PREPARE_EUROPE members reveals heterogeneity of antibiotic choice for childhood pneumonia http://ow.ly/4mIS2P

Copyright $\odot$ ERS 2016. This article is open access and distributed under the terms of the Creative Commons Attribution Non-Commercial Licence 4.0 .

\section{References}

1 Holstiege J, Schink T, Molokhia M, et al. Systemic antibiotic prescribing to paediatric outpatients in 5 European countries: a population-based cohort study. BMC Pediatrics 2014; 14: 174.

2 Kronman MP, Hersh AL, Feng R, et al. Ambulatory visit rates and antibiotic prescribing for children with pneumonia, 1994 -2007. Pediatrics 2011; 127: 411-418.

3 Grijalva CG, Nuorti JP, Griffin MR. Antibiotic prescription rates for acute respiratory tract infections in US ambulatory settings. JAMA 2009; 302: 758-766.

4 Schneider-Lindner V, Quach C, Hanley JA, et al. Secular trends of antibacterial prescribing in UK paediatric primary care. J Antimicrob Chemother 2011; 66: 424-433.

5 Lodha R, Kabra SK, Pandey RM. Antibiotics for community-acquired pneumonia in children. Cochrane Database Syst Rev 2013; 6: CD004874.

6 Harris M, Clark J, Coote N, et al. British Thoracic Society guidelines for the management of community acquired pneumonia in children: update 2011. Thorax 2011; 66: Suppl. 2, ii1-i23. 
7 Bradley JS, Byington CL, Shah SS, et al. The management of community-acquired pneumonia in infants and children older than 3 months of age: clinical practice guidelines by the Pediatric Infectious Diseases Society and the Infectious Diseases Society of America. Clin Infect Dis 2011; 53: e25-e76.

8 Esposito S, Cohen R, Domingo JD, et al. Antibiotic therapy for pediatric community-acquired pneumonia: do we know when, what and for how long to treat? Pediatr Infect Dis J 2012; 31: e78-e85.

9 Sullivan A, Edlund C, Nord CE. Effect of antimicrobial agents on the ecological balance of human microflora. Lancet Infect Dis 2001; 1: 101-114.

10 Malhotra-Kumar S, Lammens C, Coenen S, et al. Effect of azithromycin and clarithromycin therapy on pharyngeal carriage of macrolide-resistant streptococci in healthy volunteers: a randomised, double-blind, placebo-controlled study. The Lancet 2007; 369: 482-490.

11 Lode H. Safety and tolerability of commonly prescribed oral antibiotics for the treatment of respiratory tract infections. Am J Med 2010; 123: Suppl., S26-S38.

12 Infectious Diseases Society of America. Position paper: recommended design features of future clinical trials of antibacterial agents for community-acquired pneumonia. Clin Infect Dis 2008; 47: Suppl. 3, S249-S265.

13 United Nations. Composition of macro geographical (continental) regions, geographical sub-regions, and selected economic and other groupings. http://unstats.un.org/unsd/methods/m49/m49regin.htm Date last accessed: May 19, 2015. Date last updated: October 31, 2013. 